\title{
Philosophie und Medizinethik
}

\author{
Thomas Schramme
}

Online publiziert: 16. September 2016

(C) Springer-Verlag Berlin Heidelberg 2016

Was soll die Rolle der Philosophie in der Medizinethik sein? Die Tatsache, dass die Zeitschrift Ethik in der Medizin nun nach einiger Zeit wieder einmal einen Kollegen aus der Philosophie zum federführenden Schriftleiter hat, soll Anlass sein, über diese Frage zu reflektieren.

Als die Professionalisierung der Medizinethik begann, waren viele akademisch tätige PhilosophInnen an einschlägigen Debatten beteiligt - damals waren diese von den Themen Sterbehilfe und Schwangerschaftsabbruch dominiert. Die Attraktivität solcher Themen für die Moralphilosophie ergab sich unter anderem daraus, dass hier ein reales Betätigungsfeld der philosophischen Expertise vorlag. Dadurch wurden moralphilosophische Analysen nicht nur interessanter, sondern in erster Linie auch relevanter. Es bestand die Hoffnung, dass PhilosophInnen nicht nur als unnütze Kostgänger der Gesellschaft gesehen werden; eine Sorge, die sich in den letzten Jahren immer stärker in den Vordergrund drängt. Wer, wenn nicht professionelle Ethiker, sollte die Antworten auf die brennenden Fragen geben, die sich aus den modernen technischen Entwicklungen in der Medizin - damals in erster Linie die Möglichkeiten der technischen Aufrechterhaltung von vitalen Funktionen - ergaben? In der Nachfrage moralphilosophischer Expertise zeigte sich eine extern, also außerhalb der Zunft selbst, motivierte Attraktion der Medizinethik für die Philosophie.

Auch aus eher intern orientierter Motivation war die Beschäftigung mit medizinethischen Problemen attraktiv. Für die Philosophie war damit nämlich ein Weg eröffnet, die Ethik aus der abstrakten Welt der sogenannten Metaethik zu befreien. Schließlich war die Moralphilosophie der damaligen Zeit (grob gesprochen, der 60er und beginnenden 70er-Jahre des 20. Jahrhunderts) gefangen in abstrakten Debatten

Prof. Dr. T. Schramme ( $₫)$

Department of Philosophy, University of Liverpool, Mulberry Court,

Mulberry Street, Liverpool, L69 7ZY, UK

E-Mail: t.schramme@liverpool.ac.uk 
über den Status moralischer Aussagen oder die Möglichkeit moralischen Wissens. Diese Analysen hatten mehr mit der theoretischen Philosophie zu tun, als mit dem menschlichen Handeln - sie betrafen metaphysische, sprachphilosophische und erkenntnistheoretische Fragen. Ihr Erkenntniswert für wirkliche, moralische Probleme, wie sie in der Zeit der Bürgerrechtsbewegung diskutiert wurden, war bescheiden.

Alles in allem ergab sich aus dieser Gemengelage - einem gesellschaftlichen Bedürfnis nach ethischer Expertise und der Eröffnung genuin normativer Betätigungsfelder für die rein theoretisch gewordene Moralphilosophie - eine doppelte Gewinn-Situation für die philosophische Ethik. So überrascht es auch nicht, dass der Philosoph Stephen Toulmin einen berühmt gewordenen Aufsatz aus dem Jahr 1982 mit dem Titel „How medicine saved the life of ethics“ überschreiben konnte.

Was ist von dieser, für die Philosophie hoffnungsfrohen Ausgangssituation geblieben? Ich würde sagen: nicht viel. Die Gesellschaft schreit nicht gerade nach der normativen Expertise von PhilosophInnen. Wie viele akademische EthikerInnen sitzen beispielsweise im Deutschen Ethikrat? Diese Seite der hier diagnostizierten Enttäuschung von Erwartungen muss allerdings zu einem Gutteil der Philosophie selbst angekreidet werden. Ihr ist es einfach nicht gelungen, dem gesellschaftlichen Auftrag nachzukommen, relevante ethische Expertise zu vermitteln. Heute gilt das genuin philosophische Herangehen an medizinethische Fragen - von der Bewertung des Klonens zur aktuellen Debatte über personalisierte Medizin - weithin als nutzlos. Meiner Einschätzung nach liegt das an einer mangelnden Bereitschaft akademischer EthikerInnen, sich von ihrer eingeübten Perspektive zu lösen. In kurzen Worten: Philosophen wollen Probleme zerlegen, sie analysieren. Das führt zu immer kleinteiligeren Unterscheidungen. Sie wollen außerdem nicht selbst entscheiden, was das moralisch Richtige wäre, sondern die ethischen Optionen verdeutlichen. Die politische und gesellschaftliche Debatte erfordert aber das genaue Gegenteil: Die Vereinheitlichung und Vergröberung von Problemen und klare normative Bekenntnisse, bei gleichzeitiger großer Kompromissbereitschaft.

Das soll nicht heißen, dass hier, in der politischen Perspektive, eine ablehnenswerte Vereinfachung von komplexen Problemen geschieht. Es soll umgekehrt auch nicht heißen, dass dies die einzig wahre Herangehensweise darstellt und die Philosophie dabei tatsächlich nutzlos wäre. Der Punkt ist einfach, dass die genannte Ausrichtung auf gesellschaftlich weithin akzeptable Lösungen, die nicht jahrhundertelang diskutiert werden können, eben nicht den üblichen philosophischen Fokus darstellt. Insofern sind die politische und die philosophische Perspektive konträr und es überrascht nicht, dass angesichts der drängenden Probleme niemand nach der philosophischen Expertise ruft. Nun hatte ich bereits gesagt, dass sich meines Erachtens die Philosophie schuldig gemacht hat, ausschließlich auf ihrer traditionellen Perspektive zu beharren. Schließlich ist es ein Leichtes, die Suche nach der wirklich richtigen Lösung ethischer Probleme für einzig relevant zu erklären oder die Dummheit der Menschen, die das nicht sehen wollen, zu betonen. So kann man sich in Bezug auf die eigene Perspektive im Recht fühlen.

Die politische Perspektive, wie ich sie hier genannt habe, wartet natürlich nicht darauf, dass die Philosophie in die Gänge kommt. Die inzwischen erfolgte Professionalisierung der Medizinethik findet weitgehend ohne akademische Philosophen statt. In der Konsequenz hat die Philosophie damit anderen Disziplinen den Vor- 
tritt überlassen, wenn es um die Bestückung relevanter politischer Gremien geht. Es schmerzt zu sehen, dass selbst PastorInnen inzwischen im Vergleich zu PhilosophInnen als adäquatere Gesprächspartner in der gesellschaftlichen Normendebatte gesehen werden. Anscheinend gilt ein Glaubensbekenntnis heute mehr als eine kritische Analyse, die sich tastend auf den Weg der bestbegründeten Lösung macht.

Der Schritt in die richtige Richtung wäre angesichts dieser Situation, der Philosophie verschiedene Perspektiven zu eröffnen und diese zu besetzen. PhilosophInnen sollen sich natürlich nicht in PolitikerInnen verwandeln. Vielmehr sollten die genuinen Fähigkeiten der Moralphilosophie, insbesondere begriffliche Schärfe und methodologisches Bewusstsein, zielführend und variabel einsetzbar gemacht werden. Dazu müsste die philosophische Medizinethik allerdings selbst eine Methodendiskussion beginnen. Sie müsste sich beispielsweise über die Funktion der Moraltheorie in der Medizinethik verständigen. Sie müsste außerdem ihren Blick aus der Individualethik heraus auf die Politische Philosophie erweitern. Auch die nach wie vor virulente Frage, wie man Ethik überhaupt anwenden kann, gehörte mit zu dieser Methodendiskussion. Es ist allerdings sehr unwahrscheinlich, dass diese meta-theoretischen Fragen in der näheren Zukunft tatsächlich angegangen werden. Warum das so ist, hat mit der zweiten, eingangs genannten Enttäuschung zu tun.

Die erste Enttäuschung, der ich mich bisher zugewendet hatte, ergab sich aus der mangelnden Erfüllung von Ansprüchen, die an die Philosophie herangetragen wurden. Die zweite Hoffnung, die sich mit der Entwicklung der Medizinethik einstellte, war eine interne. Es schien, dass die Philosophie sich durch die anwendungsorientierten Analysen aus der selbstgewählten Enge der metaethischen Problemstellungen befreien könnte. Meiner Einschätzung nach verdankt sich die Enttäuschung dieser Hoffnung hauptsächlich der Art und Weise, wie Universitäten in Deutschland operieren. Diese institutionellen Voraussetzungen führen in der Konsequenz zu einer weithin verbreiteten Arroganz von akademisch bestallten PhilosophInnen gegenüber der sogenannten angewandten Ethik. Angesichts dieser Situation ist es auch kein Wunder, dass immer weniger jüngere, philosophisch ausgebildete Kolleginnen und Kollegen sich der Medizinethik zuwenden.

In Deutschland ist es an Universitäten nahezu ausgeschlossen, interdisziplinäre Arbeitszusammenhänge zu schaffen. Gerade die medizinischen Fakultäten sind häufig völlig losgelöst vom anderen akademischen Betrieb. Außerdem existieren natürlich immer weniger Professuren für Philosophie. Das ist dort nicht anders, als in anderen Disziplinen. Eine Ausrichtung auf die Medizinethik ist angesichts eingeschränkter Lehrkapazitäten und traditioneller Curricula ,nice to have“, aber nicht essentiell. So kann sich denn auch so manches Vorurteil, dass die Medizinethik ja gar keine richtige Philosophie sei, bestätigt sehen.

Die hier diagnostizierte doppelte Enttäuschung führt zu mancher Oberflächlichkeit in der tatsächlichen medizinethischen Debatte, wie wir sie in Deutschland vorfinden. Denn gerade der Tiefgang, die begriffliche Genauigkeit und die methodologische Sensibilität, wäre der Beitrag der Philosophie. Gibt es angesichts dieser Lage nicht doch noch einen Hoffnungsschimmer? Es dürfte Leser dieser Zeitschrift wenig verwundern, wenn ich damit schließe, den Anlass dieses Editorials, also die Bestellung meiner Person zum federführenden Schriftleiter, in diesem Zusammenhang zu nennen. Die Philosophie ist in der Akademie für Ethik in der Medizin keineswegs 
außen vor. Ja, vielleicht ist dies sogar ein Signal an die jüngere Generation philosophisch denkender Medizinethiker, unsere Zeitschrift als ihr eigenes Betätigungsfeld zu erkennen. In dieser Aussicht sehe ich einen, wenn auch schwachen, Schimmer der Hoffnung für eine philosophisch informierte Medizinethik.

Es bleibt mir der willkommene Auftrag, im Namen der Akademie für Ethik in der Medizin und des Springer Verlags Professor Gerd Richter für sein jahrelanges Engagement in der Schriftleitung, zuletzt in federführender Funktion, herzlich zu danken. Durch sein immer verbindliches und qualitätsorientiertes Wirken ist die Zeitschrift noch besser geworden. Seine kollegiale und humorvolle Art machte die Arbeit mit ihm zum Vergnügen. 